\title{
Study of the young and old compact stellar cluster population in M81
}

\author{
M. Santiago-Cortés, Y. D. Mayya and D. Rosa-González
}

Coordinación de Astrofísica, Instituto Nacional de Astrofísica, Óptica y Electrónica, Mexico

\begin{abstract}
We study the population of compact stellar clusters in M 81 using the HST/ACS images in the filters F435W, F606W and F814W that cover a total field of view of approximately 340 square arcmin, the largest area covered to date for this galaxy. We present details about the selection criteria, which were based on both morphological and photometrical features. The extracted sample of compact stellar clusters shows the presence of two cluster populations, a blue cluster group (young) with more than 300 objects, and a red cluster group (old) containing 138 objects. Surprisingly, the young group lacks clusters more massive than $10000 \mathrm{M}_{\odot}$ which are present in large numbers in its neighbor $M$ 82. The luminosity function of the young group follows a power-law distribution with an index of 2.0. The luminosity function of the red group closely resembles that of the globular clusters in the Milky Way. Assuming an age of 5 Gyr, these red clusters have masses between 0.1 and $10 \times 10^{6} \mathrm{M}_{\odot}$.
\end{abstract}

Keywords. catalogs, galaxies: individual (M 81), galaxies: spiral, galaxies: star clusters

The full poster (in pdf format) is available at http://www. astro.iag.usp.br/〜iaus266/Posters/pSantiago-Cortes.pdf. 